\title{
Neural correlates of fixation duration in natural reading: Evidence from fixation-related fMRI
}

\author{
John M. Henderson* ${ }^{1,2}$, Wonil Choi ${ }^{1}$, Steven G. Luke ${ }^{3}$, Rutvik H. Desai ${ }^{4}$ \\ ${ }^{1}$ Center for Mind and Brain \\ University of California, Davis \\ ${ }^{2}$ Department of Psychology \\ University of California, Davis \\ ${ }^{3}$ Department of Psychology \\ Brigham Young University \\ ${ }^{4}$ Department of Psychology \\ University of South Carolina
}

Abbreviated Title: Neural correlates of fixation duration in natural reading

Keywords: Reading, eye movements, fixation duration, fMRI

*Correspondence:

John M. Henderson

Center for Mind and Brain

University of California, Davis

267 Cousteau Place

Davis, CA 95618 USA

johnhenderson@ucdavis.edu 


\begin{abstract}
A key assumption of current theories of natural reading is that fixation duration reflects underlying attentional, language, and cognitive processes associated with text comprehension. The neurocognitive correlates of this relationship are currently unknown. To investigate this relationship, we compared neural activation associated with fixation duration in passage reading and a pseudo-reading control condition. The results showed that fixation duration was associated with activation in oculomotor and language areas during text reading. Fixation duration during pseudo-reading, on the other hand, showed greater involvement of frontal control regions, suggesting flexibility and task dependency of the eye movement network. Consistent with current models, these results provide support for the hypothesis that fixation duration in reading reflects attentional engagement and language processing. The results also demonstrate that fixation-related fMRI provides a method for investigating the neurocognitive bases of natural reading.
\end{abstract}




\section{Introduction}

A fundamental characteristic of skilled reading is that it unfolds spatiotemporally: The eyes move through text in a series of brief pauses (fixations) and high-velocity movements (saccades) that take the eyes from fixation to fixation [Henderson, 2013; Rayner, 1998; Rayner, 2009]. The durations of individual fixations vary during reading, with this variability providing a real-time reflection of ongoing cognitive and language processing [Rayner, 2009]. For this reason, fixation duration has served as a primary source of evidence for testing theories of reading [Breen and Clifton, 2011; Henderson, 2013; Rayner, 1998; Rayner, 2009]. Indeed, a central focus of current computational models of skilled reading is to account for the influence of text processing on fixation duration [Engbert et al., 2005; Nuthmann and Henderson, 2012; Reichle et al., 1998]. Yet little is known about the neural processes that are associated with fixation duration in reading [Henderson et al., 2014; Reichle and Reingold, 2013].

Here we use fixation-related functional magnetic resonance imaging, based on coregistered high-resolution eyetracking and fMRI, to study neural activation tied to fixation duration during natural reading. This method builds on the results of recent studies that have combined eyetracking with fMRI using arrays of words or objects (e.g., faces and buildings) in sparse formats and simplified tasks [Hillen et al., 2013; Marsman et al., 2011; Richlan et al., 2014], a technique Marsman et al. referred to as fixation-based event-related (FiBER) fMRI. In the present fixation-related fMRI study, we build on our recent study of neural correlates of fixation duration during scene perception [Henderson \& Choi, 2015], extending our investigation to natural connected-text reading. Specifically, in the present study we used fixation-related fMRI to examine the relationship between fixation duration in reading and the BOLD signal. We 
analyzed individual fixations as events and divided BOLD activation into components related to fixation onset and to fixation duration, our parametric variable of interest. In a text-reading condition, subjects read text passages in the scanner while their eye movements were recorded. We also included a pseudo-reading control condition in which subjects moved their eyes through pseudo-text that did not carry meaning but that preserved the spatial characteristics of the passages. Eye movements in pseudo-reading are similar to those in normal text reading [Henderson \& Luke, 2014; Luke and Henderson, 2013; Nuthmann and Engbert, 2009; Rayner and Fischer, 1996; Vitu et al., 1995]. Pseudo-reading therefore provides a condition for investigating reading-like eye movements while controlling for processes associated with linguistic and semantic analysis [Choi, Desai, \& Henderson, 2014; Henderson, Choi, \& Luke, 2014; Hillen et al., 2013]. Eye movements and neural activation were simultaneously recorded in both the natural text-reading and pseudo-reading conditions, and fixation-related fMRI analysis was then based on individual fixations identified in the eyetracking record.

Previous eyetracking studies have shown a tight link between cognitive processes and fixation duration, with longer fixations associated with greater attentional focus [Henderson and Ferreira, 1990; Morrison, 1984; see Rayner, 2009] and more effortful language processing [Engbert et al., 2005; Nuthmann and Henderson, 2012; Reichle et al., 1998]. However, a direct association between fixation duration and the cortical systems supporting attention and language processing during reading has not been established. Based on current reading theories, we hypothesized that fixation duration would be related to activation in brain regions typically found to be associated with attention/oculomotor control (e.g., frontal eye fields, supplementary eye fields, and intraparietal sulcus) and with language and language processing (e.g., medial and superior temporal gyri and superior temporal sulcus). We also hypothesized that frontal regions 
associated with strategic control of eye movements would likely be involved in pseudo-reading, but less so in normal text reading which would be expected to draw on more automatized eye movement control.

\section{Materials and Methods}

\section{Participants}

Twenty-nine right-handed members of the University of South Carolina community gave informed consent in accordance with the University of South Carolina Institutional Review Board. All had normal or corrected-to-normal vision and none reported reading disabilities. Data from six subjects were eliminated because of excessive movement, lack of attention (closed eyes or lack of eye movements), or poor quality eyetracking data, leaving twenty-three subjects for analysis (mean age 27.5 years, range 19-42).

\section{Materials}

Text passages (40-61 words each) were articles taken from popular online news sources, and were approximately at the $6^{\text {th }}$ to $8^{\text {th }}$ grade reading level. Word length averaged 4.96 characters (range 1-19 characters). Text passages were converted into pseudo-text using a custom font in which each letter was replaced by a geometric shape that preserved word location and word shapes but eliminated meaning [Henderson and Luke, 2012; Henderson et al., 2013; Luke and Henderson, 2013; Luke et al., 2013]. Text and pseudo-text passages were presented as multi-line paragraphs. Text content was rotated through the conditions across participants. Both 
fonts were mono-spaced, and all letters, words, and lines of text from a given passage appeared in exactly the same locations across the two fonts.

\section{Apparatus}

Stimuli were presented using an Avotec Silent Vision 6011 projector using its native resolution $(1024 \times 768)$ and a refresh rate of $60 \mathrm{~Hz}$. Passages were displayed in Courier New font with 4.76 characters subtending $1^{\circ}$ of visual angle. Eye-movements were recorded via an SR Research Eyelink 1000 long-range MRI eyetracker (spatial resolution of $0.01^{\circ}$ ) sampling at $1000 \mathrm{~Hz}$. Viewing was binocular and eye-movements were recorded from one eye.

\section{Procedure}

In each trial, text or pseudo-text appeared in paragraph form on the display and subjects were instructed to read normally and silently for comprehension in the text-reading condition and to move their eyes through the pseudo-text "as if they were reading" in the pseudo-reading condition. These instructions for the pseudo-reading task have been used extensively in the eye movement literature. Each trial lasted 12s, with an ITI of 6s between each trial, and each run included 11 text-reading and 11 pseudo-reading trials as well as 22 single-word presentation filler trials. Each participant received two functional runs and was therefore presented with 22 passages in each of the text-reading and pseudo-reading conditions. Pseudo-text passages within one run were based on text passages from the other run. Within each run, passages were presented in a random order for each participant. Each functional run lasted $15 \mathrm{~min}$.

Eye-movement Recording. Prior to each functional run, a nine-point calibration routine was used to map eye position to screen coordinates. Successful calibration required average error 
less than $.49^{\circ}$ and maximum error less than $.99^{\circ}$. A fixation cross was presented on the screen before each trial, with the first word in the text appearing at that location.

Recording of fMRI. MR data were collected on a Siemens Medical Systems 3T Trio. A 3D T1-weighted "MPRAGE" RF-spoiled rapid flash scan in the sagittal plane, and a T2/PDweighted multi-slice axial 2D dual Fast Turbo spin-echo scan in the axial plane was used. The $\mathrm{T} 1$ scans had $1 \mathrm{~mm}^{3}$ resolution and sufficient field of view to cover from the top of the head to the neck. Anatomical reference images were acquired for 8 participants with sagittal T1weighted MPRAGE $\left(\mathrm{TR}=2250 \mathrm{~ms}, \mathrm{TE}=4.18 \mathrm{~ms}\right.$, flip angle $\left.=9^{\circ}\right)$, and for 17 people with multiecho T1 $\left(\mathrm{TR}=2530 \mathrm{~ms}, \mathrm{TE} 1=1.74 \mathrm{~ms}, \mathrm{TE} 2=3.6 \mathrm{~ms}, \mathrm{TE} 3=5.46 \mathrm{~ms}\right.$, TE4 $=7.32 \mathrm{~ms}$, flip angle $\left.=7^{\circ}\right)$. For all participants, one volume of $\mathrm{T} 2 *$-weighted, gradient echo, echo-planar images ( $\mathrm{TR}=2.2 \mathrm{~s}$, $\mathrm{TE}=35 \mathrm{~ms}$, flip angle $=90^{\circ}, \mathrm{NEX}=1$ ) was collected. Volumes were composed of 36 axiallyoriented $3 \mathrm{~mm}$ slices with a $0.6 \mathrm{~mm}$ interslice gap, covering the whole brain, with FOV $=192 \mathrm{~mm}$ and $64 \times 64$ matrix, resulting in $3 \times 3 \times 3.6 \mathrm{~mm}$ voxel dimensions.

Eye Movement Data Processing. Eye-movement data were analyzed off-line to identify fixations and saccades using DataViewer (SR Research Ltd, version 1.11.1). Saccade detection used three thresholds: the eye had to move at least $.1^{\circ}$, with a velocity equal to or greater than $30 \% \mathrm{sec}$ and with an acceleration of at least $8000 \% \mathrm{sec}^{2}$. Fixation was defined as a period of time between saccades that was not part of a blink. Fixations were removed from analysis when they did not fall within a word region, or were immediately preceded or followed by a blink. This resulted in the inclusion of 21,989 fixations in the text-reading condition and 17,601 fixations in the pseudo-reading condition across all participants; on average each participant contributed 1,721 fixations across the two conditions. Basic eye movement measures were examined to ensure that participants were moving their eyes naturally during scanning and to confirm that 
fixation duration was distributed in the expected manner as required for fixation-related fMRI analysis (Table 1). Fixation duration showed variability consistent with that typically observed in text-reading and pseudo-reading studies using these materials outside the scanner [Henderson and Luke, 2012; Henderson et al., 2013; Luke and Henderson, 2013].

Table 1. Summary eye movement data. Global eye movement data for text-reading and pseudo-reading averaged over subjects. Fixation Duration = durations of all fixations; First Fixation Duration $=$ duration of the initial fixation on each word; Saccade Amplitude $=$ amplitudes of all saccades; Regression Rate $=$ percent regressive $($ backward) inter-word saccades; Skipping rate $=$ percent of words passed over and fixated later or never fixated.

\begin{tabular}{lll}
\hline Eye Movement Measure & Text-Reading & Pseudo-Reading \\
\hline Fixation Duration (ms)* & 220 & 274 \\
First Fixation Duration (ms)* & 223 & 276 \\
Saccade Amplitude (degrees)* & 2.54 & 2.46 \\
Regression Rate (percent) & 12 & 11 \\
Skipping Rate (percent)* & 39 & 45 \\
\hline
\end{tabular}

$*$ indicates a significant difference between conditions $(p<.05)$

fMRI Data Processing. The AFNI software package (Cox, 1996) was used for image analysis. Within-subject analysis involved slice timing correction, spatial co-registration (Cox and Jesmanowicz, 1999) and registration of functional images to the anatomy (Saad et al., 2009). Voxel-wise multiple linear regression was performed with the program 3dREMLfit, using reference functions representing each condition convolved with a standard hemodynamic response function. Reference functions representing the six motion parameters were included as covariates of no interest. In addition, the signal extracted from CSF was also included as noise covariates of no interest. General linear tests were conducted to obtain contrasts between conditions of interest. 
A binary regressor coding the onset of each fixation was used to examine areas associated with fixation onsets. In addition, a continuous, amplitude modulated (parametric) regressor coding the duration of each fixation was used to examine areas whose BOLD response varied with fixation duration. Thus, each fixation event was coded with two regressors: one indicating the presence/absence of a fixation, and one indicating the duration. Each TR contains multiple fixation events. However, the cumulative effects of fixations within each TR vary between TRs, due to differences in the number, duration, and intervals between the fixations. We exploited this variation to extract activations associated with high temporal resolution eye-movement parameters from low temporal resolution fMRI data. The ideal hemodynamic response resulting from these regressors was subsampled to match the time resolution of EPI images.

The individual statistical maps and the anatomical scans were projected into standard stereotaxic space (Talairach and Tournoux, 1988) and smoothed with a Gaussian filter of 5mm FWHM. In a random effects analysis, group maps were created by comparing activations against a constant value of 0 . The group maps were thresholded at voxelwise $\mathrm{p}<0.01$ and corrected for multiple comparisons by removing clusters with below-threshold size to achieve a mapwise corrected $\mathrm{p}<0.05$. Using the AFNI 3dClustSim program with 10000 iterations, the cluster threshold was determined through Monte Carlo simulations that estimate the chance probability of spatially contiguous voxels exceeding a size threshold. The smoothness of the data was estimated with the AFNI program 3dFWHMx using regression residuals as input. The analysis was restricted to a mask that excluded areas outside the brain, as well as deep white matter areas and the ventricles.

Co-Registration of Eyetracking and fMRI. The fMRI data and the eyetracking data were synchronized by aligning the onset of the experiment with the onset of the functional scan. 
Both the scanner time and the trial onset times from Experiment Builder were recorded via a dedicated TCP/IP port to a third computer running a data logger with a common clock that recorded scanner run onsets, trial onsets, and eye movement events, making it possible to identify individual fixation onsets and durations in MRI time.

\section{Results}

The analyses included a binary fixation onset regressor and a continuous fixation duration regressor. The results of the onset regressor were not of theoretical interest but are reported for completeness. The results for the onset regressor are presented in Table 2 and Figure 1.

Table 2. Activation Associated with Fixation Onset. Brain regions with activation associated with fixation onset during passage reading. Locations of peak activation are shown for each cluster with significant activity (FWE corrected at alpha $<0.05$ ). Multiple peaks required separation by a minimum of 25 voxels. The volume of the cluster $(\mu \mathrm{l})$, peak z-score, Talairach coordinates, and anatomical structures are shown. $\mathrm{L}=$ left hemisphere, $\mathrm{R}=$ right hemisphere.

\begin{tabular}{llllll}
\hline Volume & Max & $\mathrm{x}$ & $\mathrm{y}$ & $\mathrm{z}$ & Anatomical Structure \\
\hline Text-Reading & & & & & \\
76275 & 6.349 & 1 & -64 & 5 & R/L Lingual Gyrus \\
& 6.078 & 4 & 31 & 44 & R Superior Frontal Gyrus \\
& 5.837 & 7 & -52 & 47 & R Precuneus \\
& 4.936 & 28 & 49 & 23 & R Middle Frontal Sulcus \\
& 4.843 & 40 & 10 & 44 & R Middle Frontal Gyrus \\
& 4.808 & 31 & -7 & 5 & R Putamen \\
22086 & 4.607 & 31 & 19 & -3 & R Insula \\
& 4.09 & 52 & 16 & 17 & R Inferior Frontal Gyrus, Pars Opercularis \\
5022 & 5.351 & 55 & -46 & 26 & R Angular Gyrus \\
4293 & 4.41 & 37 & -73 & 23 & R Inferior Parietal Gyrus \\
& 4.19 & 52 & -28 & -6 & R Superior Temporal Sulcus \\
4077 & 4.544 & -43 & -52 & 23 & L Superior Temporal Sulcus \\
3780 & 4.831 & -25 & 49 & 20 & L Middle Frontal Sulcus/Gyrus \\
3456 & 4.656 & -37 & 22 & 35 & L Middle Frontal Gyrus \\
2754 & 5.001 & -13 & -73 & 29 & L Superior Occipital Gyrus \\
& 5.027 & -19 & -1 & 59 & L Superior Frontal Gyrus, L Frontal Eye Field \\
& 4.882 & -31 & 19 & -3 & L Insula \\
& 4.472 & -19 & -28 & 56 & L Precentral Gyrus
\end{tabular}




$\begin{array}{llllll}2160 & 5.176 & -10 & -4 & 17 & \text { L Caudate } \\ 2025 & 4.472 & 13 & -13 & 20 & \text { R Caudate } \\ 1647 & 4.644 & -37 & -76 & 26 & \text { L Angular Gyrus, L Middle Occipital Gyrus } \\ 1539 & 3.739 & -34 & -13 & 17 & \text { L Insula } \\ 1350 & 4.035 & -31 & -28 & 14 & \text { L Lat Fis-Post } \\ 756 & 4.066 & -58 & -19 & -3 & \begin{array}{l}\text { L Middle Temporal Gyrus, L Superior Temporal } \\ \text { Gyrus }\end{array}\end{array}$

Pseudo-Reading

\begin{tabular}{llllll}
89370 & 6.103 & -13 & -73 & 32 & Gyrus \\
& 5.878 & 58 & -46 & 23 & R Angular Gyrus, R Supramarginal Gyrus \\
& 5.841 & 7 & -55 & 47 & R Precuneus \\
& 5.329 & -43 & -52 & 38 & L Angular Gyrus, L Supramarginal Gyrus \\
& 5.04 & 16 & -61 & 23 & R Occipital-Parietal Sulcus, R Precuneus \\
& 4.858 & 19 & -4 & 59 & R Superior Frontal Gyru/S, R Frontal Eye Field \\
& 4.559 & -37 & -19 & 2 & L Insula \\
& 4.537 & -61 & -34 & 35 & L Supramarginal Gyrus \\
& 4.426 & 1 & -25 & 41 & R/L Middle Posterior Cingulate Gyrus \\
& 3.694 & 7 & 4 & 38 & R Middle Anterior Cingulate G/S \\
11448 & 3.682 & 37 & -76 & 29 & R Middle Occipital Gyrus \\
8505 & 4.799 & 4 & 28 & 29 & R Middle Anterior Cingulate Gyrus/Sulcus \\
& 5.04 & 31 & 55 & 17 & R Middle Frontal Gyrus \\
4806 & 4.803 & 25 & 34 & 41 & R Superior Frontal Sulcus \\
& 5.938 & 25 & -46 & -6 & R Lingual Gyrus \\
4374 & 4.869 & -22 & 1 & 59 & L Superior Frontal Gyrus/Sulcus, L Frontal Eye \\
& & & & & R Inferior Frontal Gyrus, Pars Opercularis, R \\
4293 & 3.8 & 43 & 4 & 5 & Insula \\
4023 & 4.742 & -34 & 31 & 44 & L Middle Frontal Gyrus \\
2673 & 4.346 & -22 & -43 & -6 & L Lingual Gyrus \\
2079 & 4.421 & -40 & -76 & 14 & L Middle Occipital Gyrus \\
1593 & 4.191 & 28 & -4 & -3 & R Putamen \\
756 & 4.559 & -31 & 19 & -3 & L Insula \\
729 & 3.623 & 34 & -25 & 20 & L Posterior Lateral Fissure \\
\hline
\end{tabular}




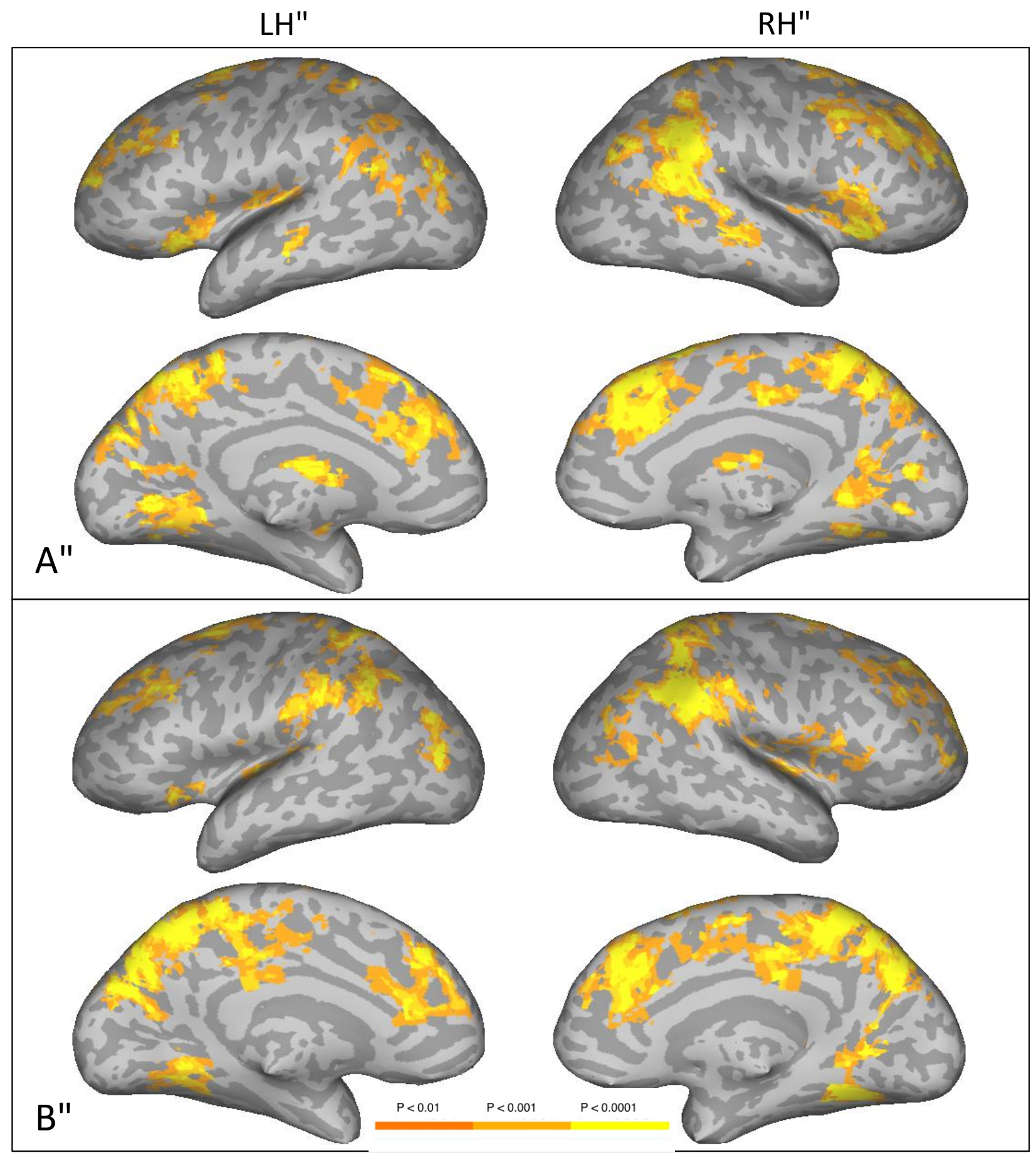

Figure 1. Areas of activation significantly correlated (FEW corrected to alpha $<0.05$ ) with fixation onset during text-reading (Panel A) and pseudo-reading (Panel B) in a whole-brain analysis. The maps are displayed using Caret [Van Essen et al., 2001] on an inflated cortical surface of a representative subject, with gyri shown as light gray and sulci shown as dark gray. 
The variable of primary theoretical interest for the fixation-related fMRI analysis was

fixation duration. In a first step, we conducted whole-brain analyses examining the associations between fMRI activation and fixation duration separately for the text-reading and pseudo-

reading conditions. The complete results of these analyses are presented in Table 3 and Figure 2.

Table 3. Activation Associated with Fixation Duration. Brain regions with activation correlated with fixation duration (FD) during passage reading. Locations of peak activation are shown for each cluster with significant activity. The volume of the cluster $(\mu 1)$, peak $\mathrm{z}$-score, Talairach coordinates, and anatomical structures are shown. $\mathrm{L}=$ left hemisphere, $\mathrm{R}=$ right hemisphere.

\begin{tabular}{llllrl}
\hline Volume & Max & x & y & z & Anatomical Structure \\
\multicolumn{2}{l}{ Text-Reading } \\
$\begin{array}{l}\text { Positive correlation } \\
17145\end{array}$ & & & & \\
& 4.339 & -4 & -67 & 8 & L Cuneus, Calcarine Sulcus, Lingual Gyrus \\
& 3.954 & 19 & -55 & 2 & R Calcarine Sulcus \\
2268 & 3.845 & -7 & 4 & 53 & L/R medial Superior Frontal Gyrus, \\
1485 & 3.937 & -55 & -34 & 8 & L Superior Temporal Gyrus/Sulcus \\
1458 & 4.017 & -43 & -1 & 44 & L Precentral Gyrus
\end{tabular}

Negative correlation

$\begin{array}{rrrrrl} & & & & & \text { R Inferior Temporal Gyrus, } \\ 6048 & -4.133 & 46 & -61 & -6 & \text { R Inferior Occipital Gyrus/Sulcus } \\ 2052 & -4.279 & -55 & -31 & 23 & \text { L Supramarginal Gyrus } \\ 1350 & -3.947 & 58 & -31 & 23 & \text { R Supramarginal Gyrus } \\ 891 & -3.764 & -40 & -64 & 2 & \text { L Middle Occipital Gyrus } \\ 864 & -3.928 & -31 & 40 & 20 & \text { L Middle Frontal Sulcus }\end{array}$

\section{Pseudo-Reading}

Positive correlation

$\begin{array}{rrrrrl}2214 & 4.753 & 49 & -58 & 41 & \text { R Angular Gyrus } \\ 2133 & 4.29 & -52 & -55 & 32 & \text { L Angular Gyrus } \\ 1701 & 4.147 & 4 & 28 & 47 & \text { R/L medial Superior Frontal Gyrus } \\ 945 & 3.655 & -13 & -73 & 38 & \text { L Precuneus } \\ 675 & 3.894 & 26 & -59 & -1 & \text { R Lingual Gyrus }\end{array}$

Negative correlation

$\begin{array}{rrrrrl}4968 & -4.445 & 43 & -67 & -3 & \text { R Middle Occipital Gyrus } \\ 3051 & -4.275 & -46 & -58 & 5 & \text { L Middle Occipital Gyrus } \\ 1836 & -4.215 & -55 & -7 & -3 & \text { L Superior Temporal Gyrus/sulcus } \\ 837 & -4.387 & 16 & -16 & -9 & \text { R Hippocampus }\end{array}$


$\begin{array}{llllll}783 & -4.734 & -49 & 10 & -12 & \text { L Superior Temporal Gyrus }\end{array}$

Text-Reading - Pseudo-Reading

Positive Correlation

$\begin{array}{rrrrrl} & & & & & \text { L Superior Temporal Gyrus, Sulcus, } \\ 2619 & 4.697 & -58 & -7 & -3 & \text { L Middle Temporal Gyrus } \\ 2376 & 3.954 & -1 & -70 & 5 & \text { L/R Lingual Gyrus, L/R Cuneus } \\ 1782 & 3.922 & -61 & -31 & 5 & \text { L Superior Temporal Gyrus } \\ 1782 & 3.632 & -46 & -13 & 44 & \text { L Precentral Gyrus } \\ & & & & & \text { L medial Superior Frontal Gyrus, } \\ 1458 & 4.625 & -4 & 7 & 53 & \text { Supplementary Eye Field } \\ 1215 & 3.849 & 52 & -7 & -6 & \text { R Superior Temporal Sulcus } \\ 891 & 4.22 & -58 & -46 & 14 & \text { L Superior Temporal Gyrus } \\ 864 & 3.839 & 16 & -52 & -3 & \text { R Lingual Gyrus } \\ 675 & 3.769 & 13 & -67 & -24 & \text { R Cerebellum }\end{array}$

Negative Correlation

\begin{tabular}{rrrrrl}
4293 & -4.607 & -52 & -52 & 32 & L Supramarginal Gyrus, Intraparietal Sulcus \\
1242 & -3.797 & -34 & 49 & 8 & L Middle Frontal Gyrus \\
999 & -3.734 & 55 & -31 & 23 & R Supramarginal Gyrus \\
999 & -4.76 & -34 & 22 & 41 & L Middle Frontal Gyrus \\
891 & -4.124 & 46 & 49 & 5 & R Middle Frontal Gyrus \\
891 & -3.678 & -10 & -70 & 50 & L Precuneus \\
\hline
\end{tabular}




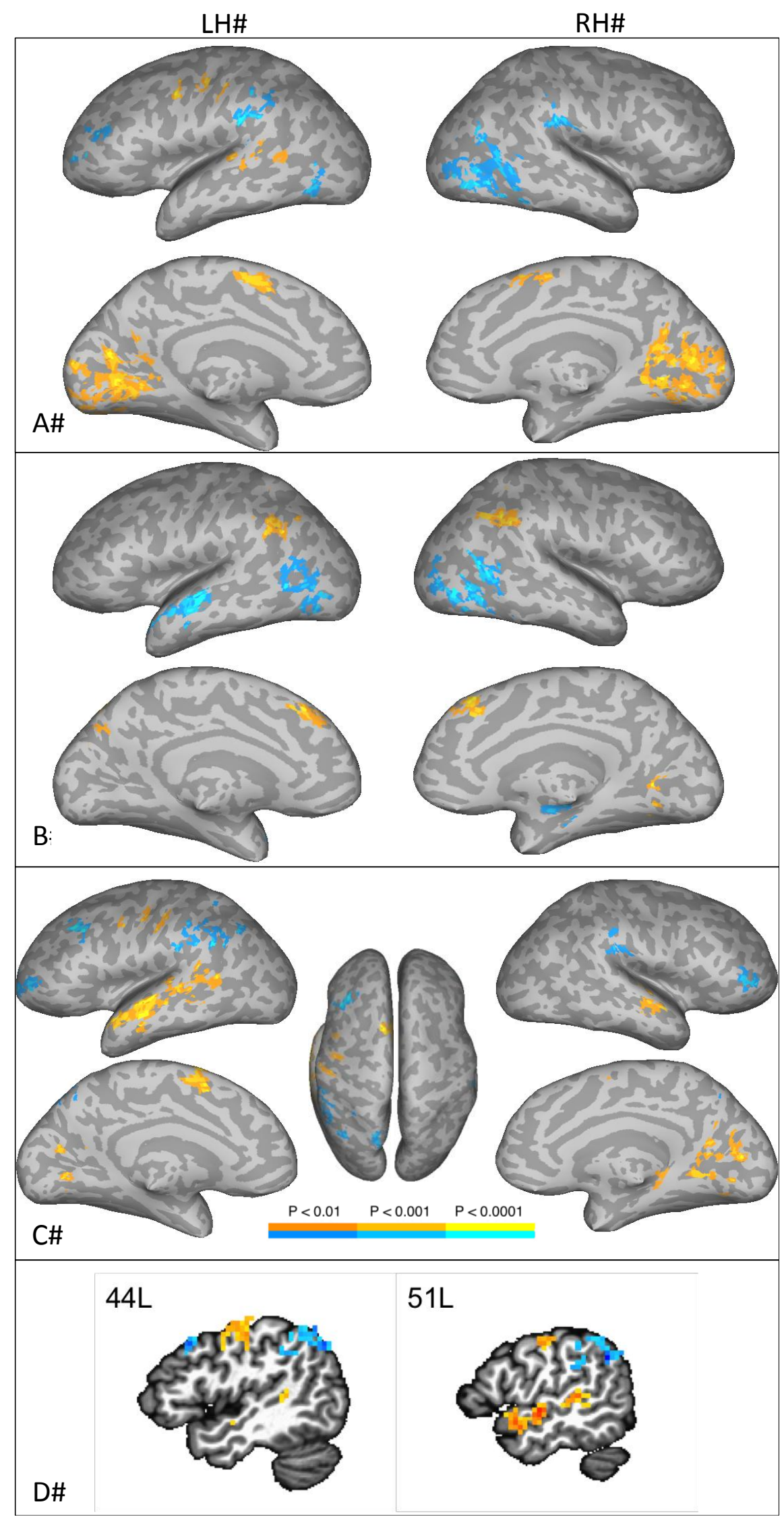


Figure 2. Areas of activation significantly correlated with fixation duration during text-reading (Panel A), pseudo-reading (Panel B), and text-reading > pseudo-reading (Panels C and D) in a whole-brain analyses. Panel D displays slices at Talairach $\mathrm{x}=-44$ and -51 . Hot regions show positive correlation and cool regions show negative correlation.

\section{Text Reading}

For text-reading, we observed positive correlations in visual areas including bilateral calcarine sulcus, cuneus, and lingual gyrus, temporal areas including left superior temporal gyrus and sulcus (STG/STS), and frontal areas involving bilateral medial superior frontal gyrus (SFG) including supplementary motor area (SMA) and the supplementary eye fields (SEF), and left precentral gyrus extending to postcentral gyrus (PoG) and to middle frontal gyrus (MFG). Textreading produced negative correlations in lateral visual areas including bilateral middle occipital gyrus (MOG) and right inferior occipital sulcus and gyrus extending to right inferior temporal gyrus, bilateral supramarginal gyrus (SMG), and left anterior middle frontal gyrus and sulcus.

\section{Pseudo Reading}

For pseudo-reading, positive correlations were observed in bilateral angular gyrus, left precuneus, and bilateral medial SFG, a region overlapping with pre-SMA. Negative correlations were observed in bilateral MOG, left anterior STG and middle STS, and right hippocampus.

\section{Text Reading vs. Pseudo reading}

Of primary theoretical interest, candidate areas for the control of fixation duration by ongoing language and cognitive processes are those that show greater correlations between fixation duration and neural activity during text-reading than pseudo-reading (Table 3 and Panels $\mathrm{C}$ and D of Figure 3). Greater positive correlations for text-reading than pseudo-reading were observed in bilateral cuneus and lingual gyrus; left temporal lobe areas along the length of STG/STS and MTG, was well as the right middle STS; left precentral gyrus extending to PoG 
and MFG; left medial SFG extending to right medial SFG and consistent with SEF; and right cerebellum.

Greater negative correlations for text-reading over pseudo-reading were observed in bilateral SMG, with left hemisphere activation extending to intraparietal sulcus (IPS); two regions of left MFG, one more dorsal area in DLPFC and the other more ventral/anterior region (consistent with BA 10); and a homologous ventral/anterior region of right MFG.

\section{Conjunction analysis of Text Reading and Pseudo reading}

A conjunction analysis of group activations for the text-reading and the pseudo-reading fixation duration activation maps was conducted to investigate which regions produced activation correlated with fixation duration in both conditions. As seen in Figure 4, voxels in the right lingual gyrus were positively correlated with fixation duration in both conditions, such that longer fixation duration was associated with more activation in this area. In comparison, activation in bilateral middle occipital and inferior temporal gyrus was negatively correlated with fixation duration in both conditions, showing that longer fixation duration was associated with less activation in these areas.

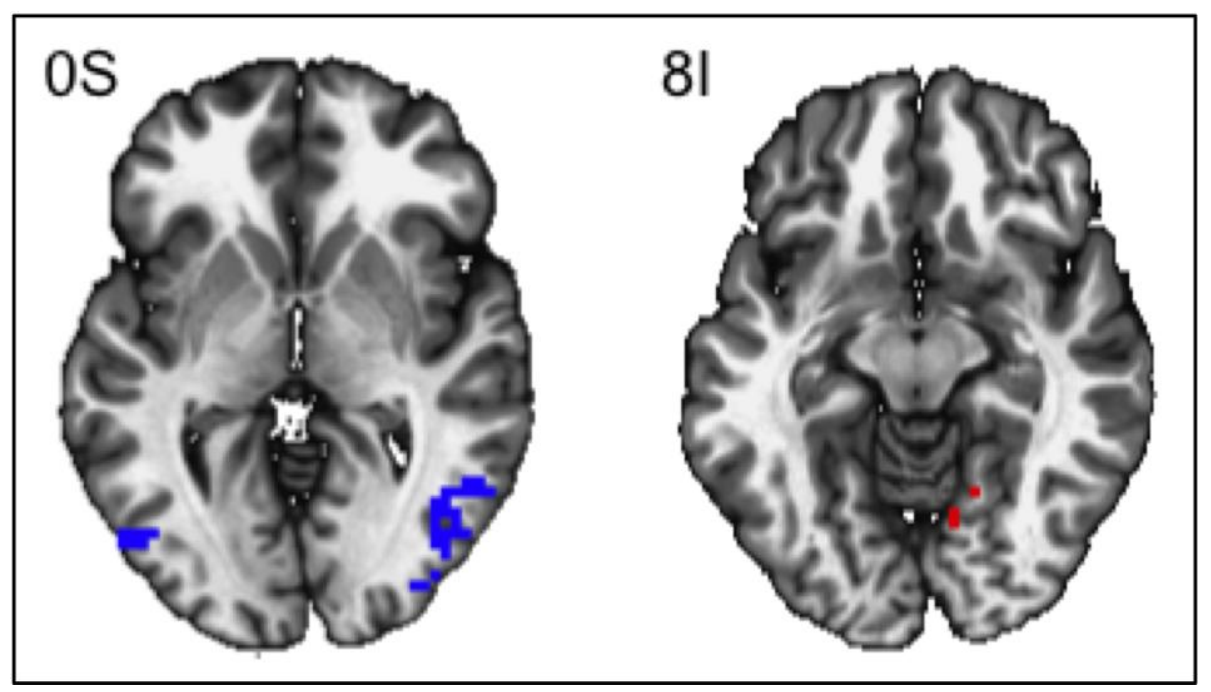


Figure 3. Areas of activation significantly correlated with fixation duration during both textreading and pseudo-reading in a conjunction analysis. The slices show the axial view of activation at Talairach $\mathrm{Z}=0$ (left panel) and $\mathrm{Z}=-8$ (right panel). Cool regions (left panel) show negative correlation and hot regions (right panel) show positive correlation.

\section{Discussion}

When we read, our eyes fixate words for varying amounts of time. Much of the variability in fixation duration is accounted for by attentional and language processes associated with the text [Rayner, 1998; Rayner, 2009]. Current theories of reading therefore attempt to account for the influence of attention and cognition on fixation duration [Engbert et al., 2005; Nuthmann and Henderson, 2012; Reichle et al., 1998]. Yet almost nothing is known about the neurocognitive systems that are related to fixation duration in reading. We addressed this topic using fixation-related fMRI, which involves co-registering high-resolution eyetracking with fMRI during natural tasks like scene perception [Henderson \& Choi, 2015] and as used in the present study, in natural reading. Comparison of natural reading with a pseudo-reading control condition allowed us to isolate cortical regions in which the association between fixation duration and fMRI activation was stronger for reading than pseudo-reading, providing a method for investigating the neural correlates of eye movements in natural reading. Using this method, we found regions of the dorsal attention and eye movement network and the ventral language network that were uniquely associated with fixation duration in natural reading.

Greater positive correlations for text-reading than pseudo-reading were observed in several areas associated with visual and attentional processing. Greater positive correlations in 
striate and peristriate regions reflected increased visual analysis of word-forms. Greater positive correlation was also clear in medial SFG in a region consistent with SEF and SMA. SEF is an attentional and eye movement control area typically found to be involved in single-step oculomotor tasks [Grosbras et al., 2005; Pierrot-Deseilligny et al., 2004; Ptak and Müri, 2013]. The observed SEF activation is interesting when contrasted with the lack of activation in another traditional eye movement area, FEF, in any of the fixation duration analyses (text-reading alone, pseudo-reading alone, or the contrast of these conditions). The lack of FEF activation in the duration-modulated analysis contrasts with the FEF-consistent activation observed bilaterally for fixation onset (Table 2 and Figure 1), as well as with recent FEF activation observed in separate (not fixation-related) study of active natural reading and scene viewing (Choi \& Henderson, 2015). It appears that activity in FEF is not directly related to modulation of fixation duration in reading. In comparison, SEF activation was related to fixation duration in text-reading alone and in the text-reading versus pseudo-reading contrast, but not in pseudo-reading alone. Given that fixation duration is related to lexical and language processing in reading, these results suggest that SEF may be involved in the interface between cognitive systems supporting text comprehension and eye movement control. As such, SEF seems to be an important component of the control network postulated by computational models of eye movement control in reading such as E-Z Reader, SWIFT, and CRISP that seek to account for fixation duration.

This profile of SEF fits well with the role of SMA in organizing complex, especially selfinitiated, movement sequences. Interestingly, SEF and surrounding SMA activation was correlated with fixation duration during text-reading but not pseudo-reading, even though the characteristics of the movements were similar. In contrast, pre-SMA activation was correlated with fixation duration in pseudo-reading rather than text-reading. The latter finding is consistent 
with the role of pre-SMA in altering movement plans or learning new sequences requiring more conscious control [Nachev et al., 2008], because pseudo-reading requires consciously controlling eye movements to simulate reading. These results indicate that in addition to the physical characteristics of the eye moments, the context (e.g., stimulus and task) in which they are made is relevant in their control. For this reason, commonly used tasks such as anti-saccade may not provide a complete picture of the neural basis of eye movements in more natural tasks such as reading. Clearly, this is an area requiring further investigation.

Greater negative correlations for text-reading than pseudo-reading were observed in a number of regions associated with attention and eye movement control. A negative correlation between fMRI activation and saccadic reaction time has previously been reported for pro- and anti-saccade tasks [Connolly et al., 2005]. In our study, several parietal regions showed negative correlations, including SMG bilaterally and more extensive left hemisphere PPC and IPS.

Bilateral PPC activation is often observed in eye movement tasks and is related to visual attention and eye movement control [Grosbras et al., 2005; Pierrot-Deseilligny et al., 2004]. IPS projects to superior colliculus (Johnston \& Everling, 2011; Paré \& Dorris, 2011; Schall, 2009), which is involved in both fixation maintenance and saccade execution (Wurtz \& Mohler, 1976; Munoz \& Wurtz, 1992). These regions therefore seem well suited as components of a network controlling the duration of fixations in reading. Activation correlated with fixation duration in left SMG is consistent with lexical control of fixation duration [Hartwigsen et al., 2010; Stoeckel et al., 2009], as proposed by E-Z Reader [Reichle et al., 1998; Reichle et al., 2003]. Right parietal lobe is typically associated with visual-spatial attention, and the dorsal inferior parietal lobule adjacent to IPS is involved in oculomotor and attentional control. Current computational theories of reading propose that covert visual-spatial attention plays an important role in eye 
movement control, with a shift of covert attention serving as a triggering event for eye movement programming [Reichle, 2011]. Greater activation may be associated with a faster shift of covert spatial attention, leading to earlier saccades and therefore shorter fixation durations.

In the direct comparison of text- vs. pseudo-reading, greater correlation of activation and fixation duration for pseudo-reading was observed in the DLPFC. This difference was driven by a negatively trending correlation for text-reading, and a positively trending correlation for pseudo-reading. DLPFC is often considered part of the eye movement control network because it is typically activated during oculomotor tasks that involve cognitive control, including memoryguided saccades and anti-saccades [Johnston and Everling, 2011; Müri and Nyffeler, 2008; Pierrot-Deseilligny et al., 2004]. It is also generally associated with executive control and working memory functions [Duncan and Owen, 2000]. We suggest that DLPFC activity increases with fixation duration in pseudo-reading to a greater extent than text-reading because pseudo-reading involves more strategic or cognitively controlled eye movements, compared to the automatized movements typical of natural reading. DLPFC projects to pre-SMA, but not to SMA [Lu et al., 1994; Luppino et al., 1993; Wang et al., 2005], and in conjunction with preSMA, likely implements controlled eye movements. Again, this finding suggests that the involvement of different components of the eye movement network is modulated by task context. In the present study we observed left but not right DLPFC activation, perhaps due to the fact that the majority of eye movements in reading and pseudo-reading (about $90 \%$ overall in the present study) are left-to-right movements.

Bilateral anterior MFG (BA 10) activation was correlated negatively with fixation duration in natural reading, but not in pseudo-reading. This region is associated with multitasking or 'cognitive branching' [Koechlin and Hyafil, 2007; Ramnani and Owen, 2004]. Words 
with shorter fixation duration may engage greater parallel processing, as text processing and planning of eye movements occur concurrently. Such concurrent processing is not required for pseudo-reading, since the stimuli do not require language processing.

In addition to effects in the attention and eye movement network, there was also clear evidence for a positive relationship between fixation duration and activation in language areas during reading of text. We observed greater activation associated with fixation duration during reading than pseudo-reading along the length of left STS/STG and MTG. Positive correlations were also observed for text but not pseudo-text in the more posterior regions of STG, STS, and MTG. Association of these regions with language processing, including phonological, semantic, syntactic, and orthographic processing is well established [Binder et al., 1997; Binder et al., 2009; Friederici, 2012; Graves et al., 2010; Price, 2010; Price, 2012; Turkeltaub et al., 2002; Vigneau et al., 2006]. As fixations increase in duration, phonological, lexical, and combinatorial semantic and syntactic processes begin to come online. The positive correlation in the precentral gyrus for text- but not pseudo-reading likely reflects increased phonological processing. This area is commonly activated in language studies with written pronounceable stimuli that overtly or covertly generate output phonology [Binder et al., 1997; Price, 2012]. These results are consistent with the common finding that lexical and higher order language processes control fixation duration in text [Rayner, 2009; Reichle et al., 1998; Reichle et al., 2003], including lexical and supra-segmental phonology [Breen and Clifton, 2011; Henderson et al., 1995; Pollatsek et al., 1992]. Stronger correlations of fixation duration and activation were seen in the contrast of text- versus pseudo-reading driven partly by negative correlations in the pseudoreading condition, especially in the left anterior STG. Because 'rest' is a highly active condition in which linguistic-semantic processes are likely active, we hypothesize that fixating 
meaningless symbols longer during pseudo-reading causes down-regulation of these processes, resulting in negative correlation in this region. We note that in the present study, differential patterns of results for text-reading and pseudo-reading are potentially due to a number of different factors at the lexical, sentential, and discourse levels of representation that cannot be dissociate in the present study, and that will be important to investigate in future work.

In summary, we have demonstrated that fixation duration in natural reading is correlated with activity in cortical structures associated with attention, oculomotor control, and language processing. These results support the hypothesis that fixation duration in reading reflects ongoing cognitive processes associated with text understanding, and with the interface of language processing, attentional systems, and eye movement control, consistent with current computational models of reading [Engbert et al., 2005; Nuthmann and Henderson, 2012; Reichle et al., 1998]. The results also suggest that the engagement of the eye movement network is flexible and task dependent. Finally, the results demonstrate that fixation-related fMRI provides a novel source of evidence for constraining theories of reading and for grounding these theories in neurobiology. This study sets the stage for using fixation-related fMRI to investigate a host of theoretical issues related to the neuro-cognition of natural reading as well as reading disabilities and deficits. 


\section{Acknowledgements}

This research was supported by the National Science Foundation (BCS-1151358) and the NIH (R01 DC010783). We thank William Brixius, Jennifer Olejarczyk, and Joseph Schmidt for their

help with data collection, Keith Rayner for his critical read of the manuscript, and Fabio Richlan and two anonymous reviewers for their comments. 


\section{References}

Binder JR, Desai RH, Graves WW, Conant LL (2009): Where is the semantic system? A critical review and meta-analysis of 120 functional neuroimaging studies. Cerebral Cortex 19:27672796.

Binder JR, Frost JA, Hammeke TA, Cox RW, Rao SM, Prieto T (1997): Human brain language areas identified by functional magnetic resonance imaging. J Neurosci 17:353-362.

Breen M, Clifton C Jr (2011): Stress matters: Effects of anticipated lexical stress on silent reading. Journal of Memory and Language 64:153-170.

Choi, W., Desai, R. H., \& Henderson, J. M. (2014). The neural substrates of natural reading: A comparison of normal and nonword text using eyetracking and fMRI. Frontiers in Human Neuroscience, 8:1024. doi: 10.3389/fnhum.2014.01024.

Choi, W, \& Henderson, JM (2015): Neural correlates of active vision: An fMRI comparison of natural reading and scene viewing. Neuropsychologia, 75, 109-115. doi:10.1016/j.neuropsychologia.2015.05.027

Connolly JD, Goodale MA, Goltz HC, Munoz DP (2005): fMRI activation in the human frontal eye field is correlated with saccadic reaction time. J Neurophysiol 94:605-611.

Duncan J, Owen AM (2000): Common regions of the human frontal lobe recruited by diverse cognitive demands. Trends Neurosci 23:475-483.

Engbert R, Nuthmann A, Richter EM, Kliegl R (2005): SWIFT: A dynamical model of saccade generation during reading. Psychological Review 112:777-813.

Friederici AD (2012): The cortical language circuit:from auditory perception to sentence comprehension. Trends Cogn Sci 16:262-268.

Graves WW, Desai R, Humphries C, Seidenberg MS, Binder JR (2010): Neural systems for 
reading aloud: A multiparametric approach. Cerebral Cortex 20:1799-1815.

Grosbras M-H, Laird AR, Paus T (2005): Cortical regions involved in eye movements, shifts of attention, and gaze perception. Hum Brain Mapp 25:140-154.

http://eutils.ncbi.nlm.nih.gov/entrez/eutils/elink.fcgi?dbfrom=pubmed\&id=15846814\&retmo de $=$ ref $\&$ cmd $=$ prlinks

Hartwigsen G, Baumgaertner A, Price CJ, Koehnke M, Ulmer S, Siebner HR (2010):

Phonological decisions require both the left and right supramarginal gyri. Proceedings of the National Academy of Sciences 107:16494-16499.

Henderson JM (2013): Eye movements. In: Reisberg, D, editor. The Oxford Handbook of Cognitive Psychology. Oxford ; New York : Oxford University Press. pp 69-82.

Henderson, JM, \& Luke, SG (2014): Stable individual differences in saccadic eye movements during reading, pseudo-reading, scene viewing, and scene search. Journal of Experimental Psychology: Human Perception and Performance 40(4): 1390-1400.

Henderson JM, Choi W, Luke SG (2014): Morphology of primary visual cortex predicts individual differences in fixation duration during text reading. Journal of Cognitive Neuroscience 26(12): 2880-2888.

Henderson, JM, \& Choi, W (2015): Neural correlates of fixation duration during real-world scene viewing: Evidence from fixation-related (FIRE) fMRI. Journal of Cognitive Neuroscience, 27(6): 1137-1145.

Henderson JM, Dixon P, Petersen A, Twilley LC, Ferreira F (1995): Evidence for the use of phonological representations during transsaccadic word recognition. Journal of Experimental Psychology: Human Perception and Performance 21:82-97.

Henderson JM, Ferreira F (1990): Effects of foveal processing difficulty on the perceptual span 
in reading: implications for attention and eye movement control. Journal of Experimental Psychology: Learning, Memory, and Cognition 16:417-429.

Henderson JM, Luke SG (2012): Oculomotor inhibition of return in normal and mindless reading. Psychonomic Bulletin \& Review 19:1101-1107.

Henderson JM, Luke SG, Schmidt J, Richards JE (2013): Co-registration of eye movements and event-related potentials in connected-text paragraph reading. Front Syst Neurosci 7.

Hillen R, Günther T, Kohlen C, Eckers C, van Ermingen-Marbach M, Sass K, Scharke W, Vollmar J, Radach R, Heim S (2013): Identifying brain systems for gaze orienting during reading: fMRI investigation of the Landolt paradigm. Frontiers in Human Neuroscience 7.

Johnston K, Everling S (2011): Frontal cortex and flexible control of saccades. In: Liversedge, SP, Gilchrist, ID, Everling, S, editors. The Oxford Handbook of Eye Movements. Oxford ; New York : Oxford University Press. pp 279-302.

Koechlin E, Hyafil A (2007): Anterior prefrontal function and the limits of human decisionmaking. Science 318:594-598.

Lu M-T, Preston JB, Strick PL (1994): Interconnections between the prefrontal cortex and the premotor areas in the frontal lobe. J Comp Neurol 341:375-392.

Luke SG, Henderson JM (2013): Oculomotor and cognitive control of eye movements in reading: Evidence from mindless reading. Atten Percept Psychophys 75:1230-1242.

Luke SG, Schmidt J, Henderson JM (2013): Temporal Oculomotor Inhibition of Return and Spatial Facilitation of Return in a Visual Encoding Task. Frontiers in Psychology 4.

Luppino G, Matelli M, Camarda R, Rizzolatti G (1993): Corticocortical connections of area F3 (SMA-proper) and area F6 (pre-SMA) in the macaque monkey. J Comp Neurol 338:114140. 
Marsman JBC, Renken R, Velichkovsky BM, Hooymans JMM, Cornelissen FW (2011):

Fixation based event-related fmri analysis: Using eye fixations as events in functional magnetic resonance imaging to reveal cortical processing during the free exploration of visual images. Hum Brain Mapp 33:307-318.

Morrison RE (1984): Manipulation of stimulus onset delay in reading: evidence for parallel programming of saccades. Journal of Experimental Psychology: Human Perception and Performance 10:667-682.

Müri RM, Nyffeler T (2008): Neurophysiology and neuroanatomy of reflexive and volitional saccades as revealed by lesion studies with neurological patients and transcranial magnetic stimulation (TMS). Brain Cogn 68:284-292.

http://www.eric.ed.gov/ERICWebPortal/recordDetail?accno=EJ819433.

Nachev P, Kennard C, Husain M (2008): Functional role of the supplementary and presupplementary motor areas. Nat Rev Neurosci 9:856-869.

Nuthmann A, Engbert R (2009): Mindless reading revisited: An analysis based on the SWIFT model of eye-movement control. Vision Research 49:322-336.

Nuthmann A, Henderson JM (2012): Using CRISP to model global characteristics of fixation durations in scene viewing and reading with a common mechanism. Visual Cognition 20:457-494.

Pierrot-Deseilligny C, Milea D, Müri RM (2004): Eye movement control by the cerebral cortex. Curr Opin Neurol 17:17-25.

Pollatsek A, Lesch M, Morris RK, Rayner K (1992): Phonological codes are used in integrating information across saccades in word identification and reading. Journal of Experimental Psychology: Human Perception and Performance 18:148-162. 
Price CJ (2010): The anatomy of language: a review of 100 fMRI studies published in 2009.

Annals of the New York Academy of Sciences 1191:62-88.

Price CJ (2012): A review and synthesis of the first 20years of PET and fMRI studies of heard speech, spoken language and reading. NeuroImage 62:816-847.

Ptak R, Müri RM (2013): The parietal cortex and saccade planning: lessons from human lesion studies. Frontiers in Human Neuroscience 7:254.

Ramnani N, Owen AM (2004): Anterior prefrontal cortex: insights into function from anatomy and neuroimaging. Nat Rev Neurosci 5:184-194.

Rayner K (1998): Eye movements in reading and information processing: 20 years of research. Psychological Bulletin 124:372-422.

Rayner K, Fischer MH (1996): Mindless reading revisited: eye movements during reading and scanning are different. Perception \& Psychophysics 58:734-747.

Rayner K (2009): The 35th Sir Frederick Bartlett Lecture: Eye movements and attention in reading, scene perception, and visual search. The Quarterly Journal of Experimental Psychology 62:1457-1506.

http://eutils.ncbi.nlm.nih.gov/entrez/eutils/elink.fcgi?dbfrom=pubmed\&id=19449261\&retmo de $=$ ref $\&$ cmd $=$ prlinks

Reichle ED (2011): Serial-attention models of reading. The Oxford Handbook of Eye Movements:767-786.

Reichle ED, Pollatsek A, Fisher DL, Rayner K (1998): Toward a model of eye movement control in reading. Psychological Review 105:125-157.

Reichle ED, Rayner K, Pollatsek A (2003): The E-Z reader model of eye-movement control in reading: comparisons to other models. Behav Brain Sci 26:445-476. 
Reichle ED, Reingold EM (2013): Neurophysiological constraints on the eye-mind link.

Frontiers in Human Neuroscience 7:361-361.

Richlan F, Gagl B, Hawelka S, Braun M, Schurz M, Kronbichler M, Hutzler F (2014): Fixationrelated fMRI analysis in the domain of reading research: Using self-paced eye movements as markers for hemodynamic brain responses during visual letter string processing. Cerebral Cortex in press.

Stoeckel C, Gough PM, Watkins KE, Devlin JT (2009): Supramarginal gyrus involvement in visual word recognition. Cortex 45:1091-1096.

Turkeltaub PE, Eden GF, Jones KM, Zeffiro TA (2002): Meta-Analysis of the Functional Neuroanatomy of Single-Word Reading: Method and Validation. NeuroImage 16:765-780.

Van Essen DC, Drury HA, Dickson J, Harwell J, Hanlon D, Anderson CH (2001): An integrated software suite for surface-based analyses of cerebral cortex. J Am Med Inform Assoc 8:443459.

Vigneau M, Beaucousin V, Hervé PY, Duffau H, Crivello F, Houdé O, Mazoyer B, TzourioMazoyer N (2006): Meta-analyzing left hemisphere language areas: Phonology, semantics, and sentence processing. NeuroImage 30:1414-1432.

Vitu F, O'Regan JK, Inhoff AW, Topolski R (1995): Mindless reading: eye-movement characteristics are similar in scanning letter strings and reading texts. Perception \& Psychophysics 57:352-364.

Wang Y, Isoda M, Matsuzaka Y, Shima K, Tanji J (2005): Prefrontal cortical cells projecting to the supplementary eye field and presupplementary motor area in the monkey. Neurosci Res 53:1-7. 
Highlights for NeuroImage

Fixation duration (FD) reflects cognitive processes in natural reading

The neurocognitive correlates of this relationship are currently unknown

We investigated this relationship using fixation-related (FIRE) fMRI

We compared FDs in reading and a pseudo-reading control

Results supports hypothesis that FD reflects attention and language processing 